Received: 10 August 2017

Accepted: 8 March 2018

Published online: 12 April 2018
CIENTIFIC REP

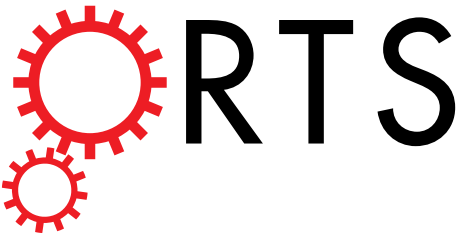

OPEN

\section{The C-terminal HRET sequence of Kv1.3 regulates gating rather than targeting of Kv1.3 to the plasma} membrane

\author{
Orsolya Voros ${ }^{1}$, Orsolya Szilagyi ${ }^{1}$, András Balajthy ${ }^{2}$, Sándor Somodi ${ }^{3}$, Gyorgy Panyi ${ }^{4}$ \& \\ Péter Hajdu ${ }^{1,5}$
}

Kv1.3 channels are expressed in several cell types including immune cells, such as T lymphocytes. The targeting of Kv1.3 to the plasma membrane is essential for T cell clonal expansion and assumed to be guided by the C-terminus of the channel. Using two point mutants of Kv1.3 with remarkably different features compared to the wild-type Kv1.3 (A413V and H399K having fast inactivation kinetics and tetraethylammonium-insensitivity, respectively) we showed that both Kv1.3 channel variants target to the membrane when the C-terminus was truncated right after the conserved HRET sequence and produce currents identical to those with a full-length C-terminus. The truncation before the HRET sequence (NOHRET channels) resulted in reduced membrane-targeting but non-functional phenotypes. NOHRET channels did not display gating currents, and coexpression with wild-type Kv1.3 did not rescue the NOHRET-A413V phenotype, no heteromeric current was observed. Interestingly, mutants of wildtype Kv1.3 lacking HRET(E) (deletion) or substituted with five alanines for the HRET(E) motif expressed current indistinguishable from the wild-type. These results demonstrate that the C-terminal region of Kv1.3 immediately proximal to the $\mathbf{S} 6$ helix is required for the activation gating and conduction, whereas the presence of the distal region of the C-terminus is not exclusively required for trafficking of Kv1.3 to the plasma membrane.

Potassium channels are essential players in setting the membrane potential and in the regulation of intracellular signaling in both excitable and non-excitable cells $s^{1,2}$. Voltage-gated potassium channels of the large family of $\mathrm{K}^{+}$ channels (Kv channels) are comprised of four subunits (both hetero- and homomers) in native cells and heterologous expression systems. A Kv channel subunit consists of six $\alpha$-helical transmembrane segments (S1-S6). The intracellular N-terminal region of the channel contains the tetramerization T1 domain, which is required for assembly of individual subunits in the ER. Furthermore, accessory $\mathrm{Kv} \beta$ subunits can bind to the $\mathrm{N}$ terminus, and enable the binding of several signaling molecules, such as kinases ${ }^{3}$. The highly-conserved pore region of $\mathrm{Kv}$ channels is formed by the linker between the S5 and S6, and mainly functions as a selectivity filter for $\mathrm{K}^{+}$ions. The fourth transmembrane segment, which contains several positively charged amino acid residues, is considered to be the voltage sensor of all $\mathrm{Kv}$ channels ${ }^{4}$. The $\mathrm{C}$-terminus of the channel can be coupled to various linker/adaptor proteins, which can anchor the protein to the cytoskeleton, bind to kinases or even regulate steering of the channels to the plasma membrane ${ }^{5-10}$.

Several studies have been published on the birth, membrane trafficking/targeting and assembly of channels ${ }^{1,2}$. During translation of the channel mRNA, the nascent polypeptide chain is embedded into the ER membrane, from which the balance between the retrograde and anterograde transport rates determines the expression level

${ }^{1}$ Department of Biophysics and Cell Biology, Faculty of Medicine, University of Debrecen, 400, 1 Egyetem sq., Debrecen, 4032, Hungary. ${ }^{2}$ Department of Pediatrics, Faculty of Medicine, University of Debrecen, 400, 1 Egyetem sq., Debrecen, 4032, Hungary. ${ }^{3}$ Department of Internal Medicine, Faculty of Medicine, University of Debrecen, 400, 1 Egyetem sq., Debrecen, 4032, Hungary. ${ }^{4}$ Department of Biophysics and Cell Biology, Faculty of Medicine, University of Debrecen, 1 Egyetem sq., 4032, Hungary. MTA-DE-NAP B Ion Channel Structure-Function Research Group, RCMM, University of Debrecen, 400, Debrecen, Hungary. ${ }^{5}$ Department of Biophysics and Cell Biology, Faculty of Dentistry, University of Debrecen, 400, 1 Egyetem sq., Debrecen, 4032, Hungary. Correspondence and requests for materials should be addressed to P.H. (email: hajdup@med.unideb.hu) 
in the plasma membrane. Though many membrane proteins have a cleavable signaling sequence for targeting to the plasma membrane, Kv1 channels lack this motif and the S2 segment serves as a recognition site for targeting ${ }^{1}$. Other protein motifs were described in Kv1 channels that facilitate retention in the ER or forward targeting. For Kv1.4 channels the "VXXSL" motif of the intracellular C-terminus promotes high surface expression". The pore region of Kv1.4 channels also governs targeting to the membrane. However, the Kv1.1 channel lacks the "VXXSL" sequence, instead it possesses the "HRET" amino-acid motif right after the S6 segment in the C-tail. Introduction of a stop codon after the $\mathrm{R}$ or $\mathrm{H}$ residues of this latter sequence leads to a loss of $\mathrm{K}^{+}$conduction without altering the cell surface expression level ${ }^{12}$. Lu et al. showed that Shaker $\mathrm{K}^{+}$channels, a Kv1 analogue in Drosophila, are also targeted to the plasma membrane without the "HRE" region of the C-terminal. The lack of the "HRE" region in Shaker resulted in a drastic change in the steady-state gating parameters ${ }^{13}$, as opposed to the loss of the conductance as in Kv1.1. On the contrary, deletion of amino acids preceding the "HRET" sequence in Shaker, (still part of the C-terminal region) prevented channel expression. Kv1.3 was shown to be guided to the cell membrane by the presence of two acidic glutamate (E) residues at the C-terminus of the channel: removal of these amino acids with C-terminal deletion or mutation to isoleucine resulted in the loss of ionic current and plasma membrane expression $^{14}$.

Kv1.3 channels are present in various cell types including T cells, where they play an important role in controlling $\mathrm{Ca}^{2+}$ signaling upon antigen-induced activation. Two papers demonstrated recently that truncation of the Kv1.3 C-terminus can vastly deteriorate the anterograde transport of the channels ${ }^{14,15}$. It is well documented that the $\mathrm{C}$-terminus of $\mathrm{Kv}$ channels is an important interaction site for several scaffolding proteins (PSD95, SAP-97, cortactin) ${ }^{6,16-20}$. Moreover, the "HRET" sequence in Kv1.3 channels, which is right after the $\mathrm{S} 6$ segment, is close to the activation gate ${ }^{21}$. Hence, truncation of the C-terminal including this motif may interfere with the activation gate and results in cell-surface targeting but a non-conducting construct. Consequently, the objective of this study was to clarify whether the removal of the major part of the C-terminal tail of Kv1.3 - with or without the HRET(E)-region - has any impact on the cell-surface trafficking or/and channel conductance of Kv1.3.

\section{Results}

Strategic considerations for designing the Kv1.3 constructs. Three strategies were applied for unique identification of the Kv1.3 subunits transfected into HEK or CHO cells. First, we introduced the A413V mutation in the S6, which has been shown to accelerate dramatically the inactivation kinetics of A413V homomers as compared to wild-type channels $\left(\tau_{\mathrm{A} 413 \mathrm{~V}}=4 \mathrm{~ms}, \tau_{\mathrm{WT}}=200 \mathrm{~ms}\right.$, Fig. $1 \mathrm{~A}$ and $\left.\mathrm{C}^{22,23}\right)$. As it was shown earlier, heterotetramers consisting of $\mathrm{A} 413 \mathrm{~V}$ and the WT subunits display intermediate inactivation kinetics between $\tau_{\mathrm{A} 413 \mathrm{~V}}$ and $\tau_{\mathrm{WT}}$ depending on the number of mutant subunits in the tetrameric channel ${ }^{22,24,25}$ (also see Suppl. Figure 1). Consequently, the presence of heteromeric channels can be easily tracked in the membrane by fitting the inactivation kinetics of the $\mathrm{K}^{+}$current $^{23}$.

Second, we substituted histidine $(\mathrm{H})$ for lysine $(\mathrm{K})$ at residue 399 which resulted in a TEA (tetraethylammonium)- insensitive, phenotype, as described earlier (Fig. 1A and C $)^{26-28}$. The assembly of TEA sensitive $\left(\mathrm{K}_{\mathrm{d}}\right.$ $\approx 11.4 \mathrm{mM})$ and insensitive $\left(\mathrm{K}_{\mathrm{d}} \approx 2000 \mathrm{mM}\right) \mathrm{Kv} 1.3$ subunits modifies the affinity of the heterotetramer for TEA, hence the heteromultimer formation can be easily identified by the application of $100 \mathrm{mM} \mathrm{TEA}^{26}$.

Third, for immunocytochemistry experiments we used constructs that had the FLAG insert in the extracellular loop between the S1 and S2 segments. The insertion of the FLAG epitope does not alter dramatically the properties of Kv1.3 as shown earlier but allows labeling of the channels expressed in the plasma membrane.

These mutations were combined with various truncations/mutations in the C-terminus and fluorescence protein tagging on the N-terminus: EGFP for all constructs except the WT channel in the co-expression experiments, where mCherry was used. Truncations at the C-terminus were generated by introducing a stop codon after (constructs denoted with $\triangle \mathrm{C}$ ) or before the "HRET" sequence (labeled with NOHRET ending) (Fig. 1B).

Deletion of the C-terminal region does not reduce current of $\Delta C$ channels. Several studies reported that the removal of the $\mathrm{C}$-terminus of $\mathrm{Kv}$ channels drastically influences their cell membrane expression, and that the "HRET" sequence is crucial for anterograde trafficking ${ }^{12}$. To test this scenario for Kv1.3 we used the A413V- $\triangle \mathrm{C}$ and A413V-NOHRET constructs (Fig. 1B) and expressed them in HEK and CHO cell lines. The $\triangle \mathrm{C}$ nomenclature in this paper refers to a construct that is truncated right after the HRET motif $(\triangle 440-523)$ whereas NOHRET indicates the construct lacks the entire C-terminus including the HRET motif as well $(\Delta 436-523)$. Figure $2 \mathrm{~A}$ shows that expression of the A413V- $\Delta \mathrm{C}$ construct results in a robust whole-cell current having fast inactivation kinetics, as it was seen for full-length homotetrameric A413V (Fig. 1C). On the contrary, HEK transfected under identical conditions with the A413V-NOHRET construct did not show any voltage- and time-dependent current. Comparison of the current density (CD, peak current at $+50 \mathrm{mV}$ divided by the whole-cell capacitance) for A413V-FL, A413V- $\Delta$ C and A413V-NOHRET in HEK cells (Fig. 2C) showed that the $\triangle \mathrm{C}$-truncated construct had a slightly higher median $\mathrm{CD}$ than the full-length one although the difference was not statistically significant $\left(\mathrm{p}=0.053, \mathrm{CD}_{\mathrm{FL}}=0.26 \pm 0.05 \mathrm{nA} / \mathrm{pF}(\mathrm{n}=13, \mathrm{~A} 413 \mathrm{~V}-\mathrm{FL}), \mathrm{CD}_{\Delta \mathrm{C}}=0.71 \pm 0.15 \mathrm{nA} /\right.$ $\mathrm{pF}(\mathrm{n}=15, \mathrm{~A} 413 \mathrm{~V}-\Delta \mathrm{C}))$ whereas the CD for the A413V-NOHRET is $\mathrm{CD}_{\text {NOHRET }}=0.016 \pm 0.007 \mathrm{nA} / \mathrm{pF}(\mathrm{n}=4)$, $\mathrm{p}<0.05$. To rule out the influence of the expression system on our results (e.g., the presence of endogenous Kv1.x channels/subunits in HEK, which may facilitate the forward trafficking of heteromers of WT and A413V- $\Delta \mathrm{C}$ mutant subunits to the membrane, or cell-specific protein sorting and trafficking) the whole set of experiments was repeated in $\mathrm{CHO}$ cells ${ }^{29,30}$. CHO cells do not show measurable whole-cell outward current at $+50 \mathrm{mV}$ (Suppl. Figure 2) and thus, are suitable for our experiments. The results in $\mathrm{CHO}$ were qualitatively similar to the ones obtained in HEK, the A413V-FL and the A413V- $\Delta \mathrm{C}$ constructs gave rise to A413V homotetrameric currents of similar density whereas practically zero current could be recorded following the transfection with 
A

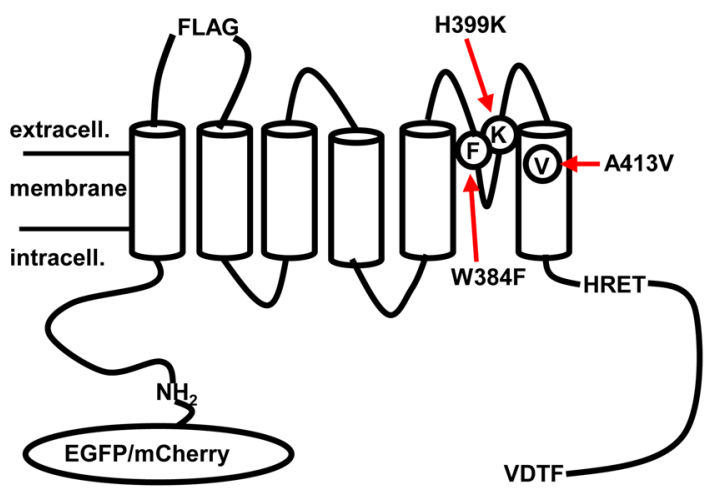

B

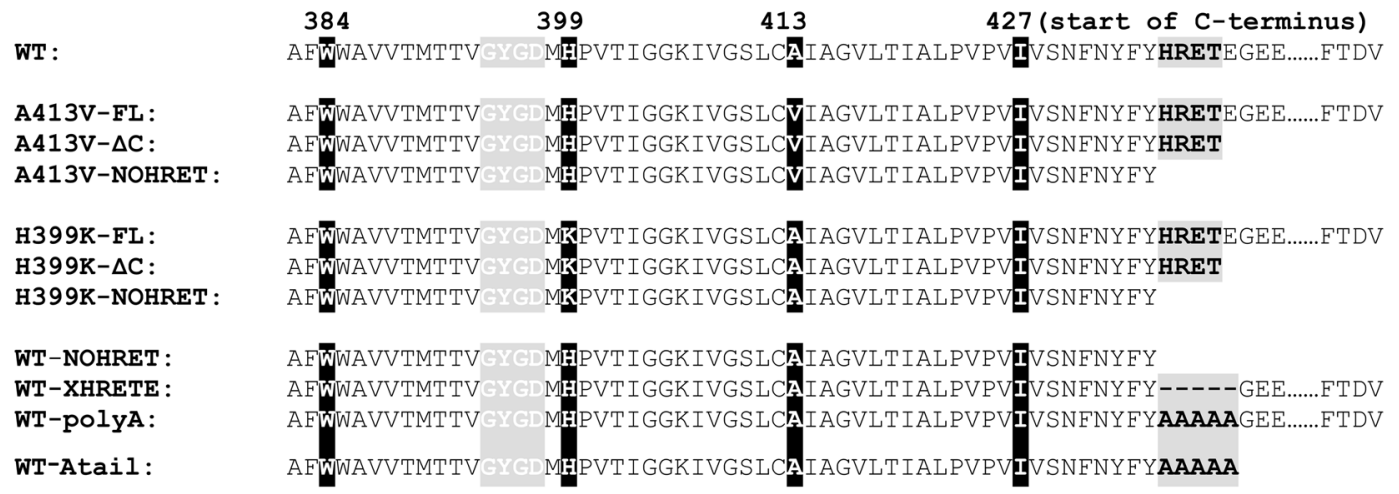

C
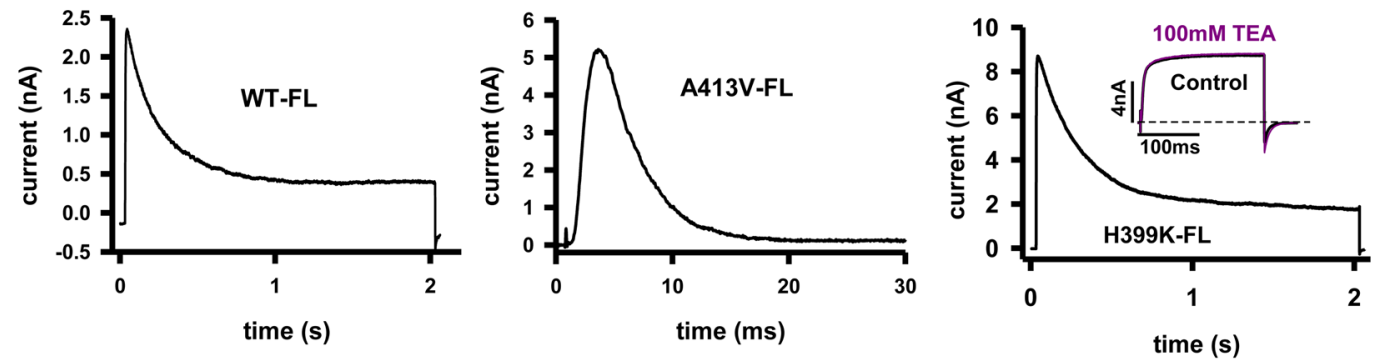

Figure 1. Point- and C-terminal deletion mutants of Kv1.3 channels. (A) Schematic illustration of wild type (WT) Kv1.3 pore-forming alpha subunit with N-terminal EGFP/mCherry, FLAG epitope, point mutations (H399K, A413V, W384F), the HRET sequence and the last four amino acids of C-terminus (FTDV). (B) Amino acid sequences of WT Kv1.3 and mutant Kv1.3 s starting at amino acid 382 (“....." represents rest of the C-terminus not relevant to this study). Amino acids are listed by their one letter designations, FL: full-length C-terminus, $\triangle \mathrm{C}$ : C-terminal truncated at 439, NOHRET: truncation after postion 435. XHRETE: deletion mutant removing HRETE sequence. polyA: replacement HRETE with AAAAA, Atail: HRETE changed for AAAAA, and rest of C-terminus is removed. Starting amino acid of C-terminus 427 is indicated. (C) Current traces of WT (mCherry tagged, left), A413V-FL (center) and H399K-FL (right) channels expressed in HEK cells. WT (outside-out patch configuration): cells were depolarized to $+50 \mathrm{mV}$ from a holding potential of $-120 \mathrm{mV}$ for 2 seconds. A413V-FL (whole-cell configuration): cells were kept at $-120 \mathrm{mV}$ then depolarized to $+50 \mathrm{mV}$ for $30 \mathrm{~ms}$. H399K (whole-cell configuration): cells were depolarized to $+50 \mathrm{mV}$ from $-120 \mathrm{mV}$ for 2 seconds. Inset: whole-cell current of a cell expressing H399K-FL and depolarized to $0 \mathrm{mV}$ from a holding potential of $-120 \mathrm{mV}$ for $200 \mathrm{~ms}$ in the presence and absence of $100 \mathrm{mM}$ TEA.

the A413V-NOHRET construct (Fig. 2D, $\mathrm{CD}_{\mathrm{FL}}=0.36 \pm 0.1 \mathrm{nA} / \mathrm{pF}(\mathrm{n}=5, \mathrm{~A} 413 \mathrm{~V}-\mathrm{FL}), \mathrm{CD}_{\Delta \mathrm{C}}=0.32 \pm 0.09 \mathrm{nA} / \mathrm{pF}$ $\left.(\mathrm{n}=5, \mathrm{~A} 413 \mathrm{~V}-\Delta \mathrm{C}), \mathrm{p}=0.748, \mathrm{CD}_{\text {NOHRET }}=0.008 \pm 0.002 \mathrm{nA} / \mathrm{pF}(\mathrm{n}=10), \mathrm{p}<0.05\right)$. Due to the lack of whole-cell currents in untransfected $\mathrm{CHO}$ we used these cells in our subsequent experiments. 
A

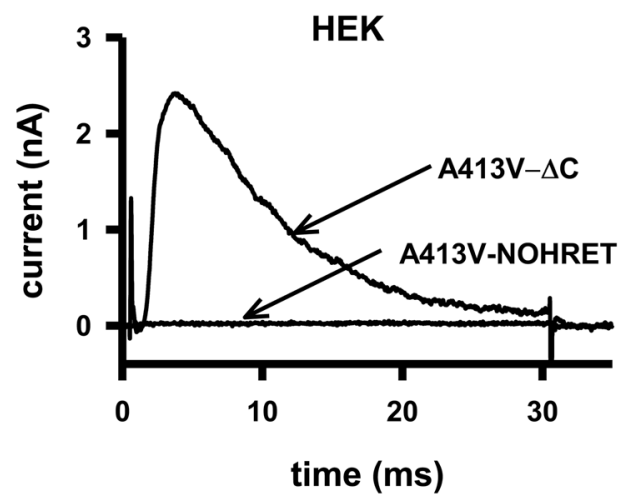

B

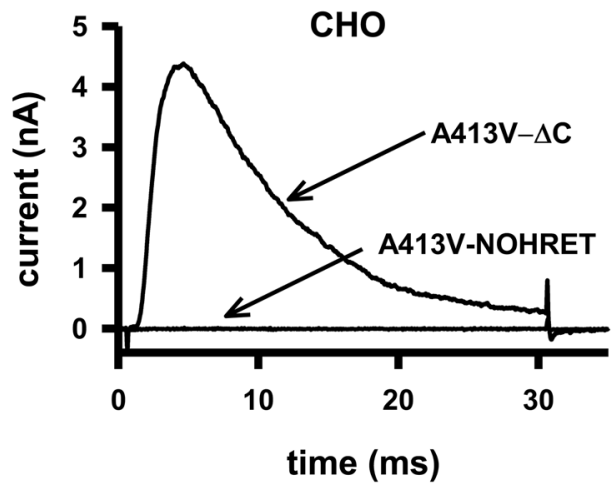

C

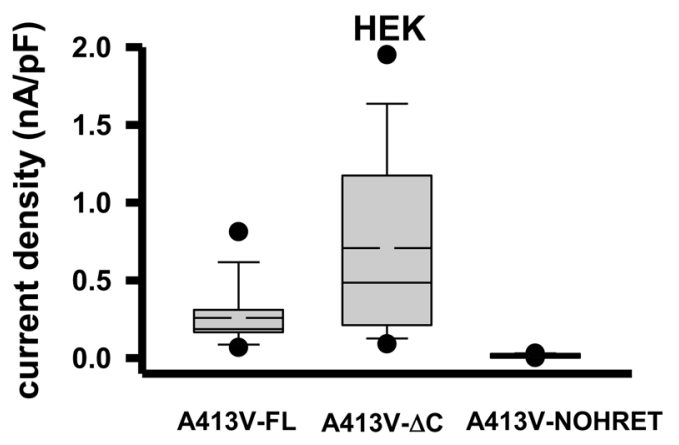

D

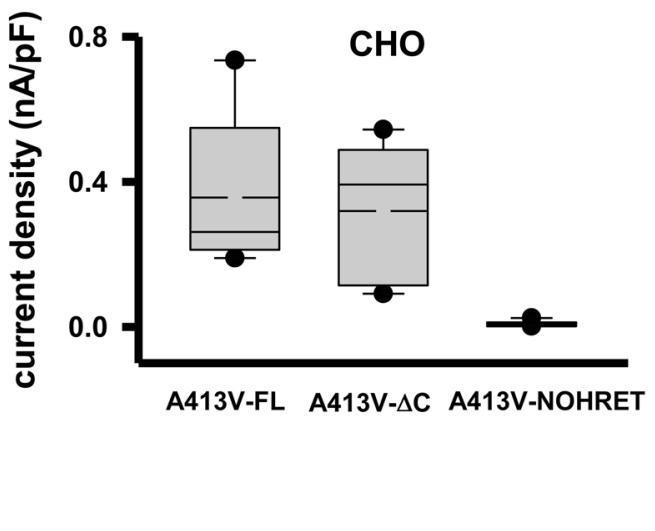

Figure 2. Current of A413V truncated mutants in HEK and CHO cells. (A) Representative current traces recorded in HEK transfected with the EGFP-tagged A413V- $\Delta \mathrm{C}$ or the A413V-NOHRET construct (arrows). Currents were recorded upon 30-ms-long depolarizations to $+50 \mathrm{mV}$ from a holding potential of $-120 \mathrm{mV}$. (B) Representative current traces recorded in CHO transfected with the EGFP-tagged A413V- $\Delta \mathrm{C}$ or the A413V-NOHRET construct. Recording conditions were the same as in panel A. (C,D) Box plot of the current density measured in HEK (C) or CHO (D) transfected with the full length (A413V-FL), A413V- $\Delta$ C or A413VNOHRET constructs. The data are reported as mean (dashed line), median (solid line), first (top box) and third quartiles (bottom box), and maximum and minimum. Current density was determined as detailed in the materials and methods.

We also tested whether all these channel constructs are properly translated by the cells using western-blot against a FLAG epitope inserted between the S1-S2 helical segments of all the constructs (Fig. 1A). Suppl. Figure 3 illustrates that $\mathrm{CHO}$ cells transfected with any of the three FLAG-tagged constructs express EGFP-tagged Kv1.3 channel subunit of appropriate size (between $85-95 \mathrm{kDa}$ ).

Pharmacology reveals homomers of $\mathrm{H} 399 \mathrm{~K}-\Delta \mathrm{C}$ channels in the membrane. To rule out mutationspecific effects (e.g. A413V) on the expression of the $\mathrm{K}^{+}$current, we used an alternative, pharmacological approach to learn if carboxyl-terminal deleted channels can reach the plasma membrane. TEA, a general inhibitor of various $\mathrm{K}^{+}$channels, is a fast open-channel blocker and its affinity depends on an aromatic amino-acid side chain in the extracellular mouth of the pore region ${ }^{27,31}$. Previously we showed that in Kv1.3 the mutation of the TEA-binding residue (H399) to a positively charged amino acid (e.g. lysine, K) led to a completely TEA-insensitive channel ${ }^{26}$. Figure 3A shows currents of the FLAG-H399K-FL (full-length subunits with FLAG tag) mutant in $\mathrm{CHO}$ cells in control extracellular solution and in the presence of $100 \mathrm{mM}$ TEA, the overlapping currents indicate the lack of inhibition ( $\mathrm{RF}=0.95 \pm 0.01, \mathrm{n}=5$, see Materials and Methods). When CHO cells were transfected with the FLAG-H399K- $\Delta$ C plasmid we measured currents similar to the full-length $\mathrm{H} 399 \mathrm{~K}$ construct (Fig. 1C and Suppl. Figure 4A) and also displayed TEA resistance (Fig. 3B, RF $=0.98 \pm 0.01, n=5$ ). The C-terminal deletion that includes the "HRET" motif as well (FLAG-H399K-NOHRET) resulted in a phenotype that lacks outward $\mathrm{K}^{+}$current, just as we described for the A413V mutant (Suppl. Figure 4B).

In summary, using either kinetically tagged (A413V) or pharmacologically tagged (H399K) C-terminally truncated Kv1.3 we demonstrated that C-terminal truncated subunits (A413V- $\Delta \mathrm{C}$ or H399K- $\Delta \mathrm{C}$ ) form functional homotetrameric channels in the membrane regardless of the expression system. However, we were unable to record whole-cell currents when the C-terminal truncated constructs that lack the "HRET" motif as well (i.e. 
A
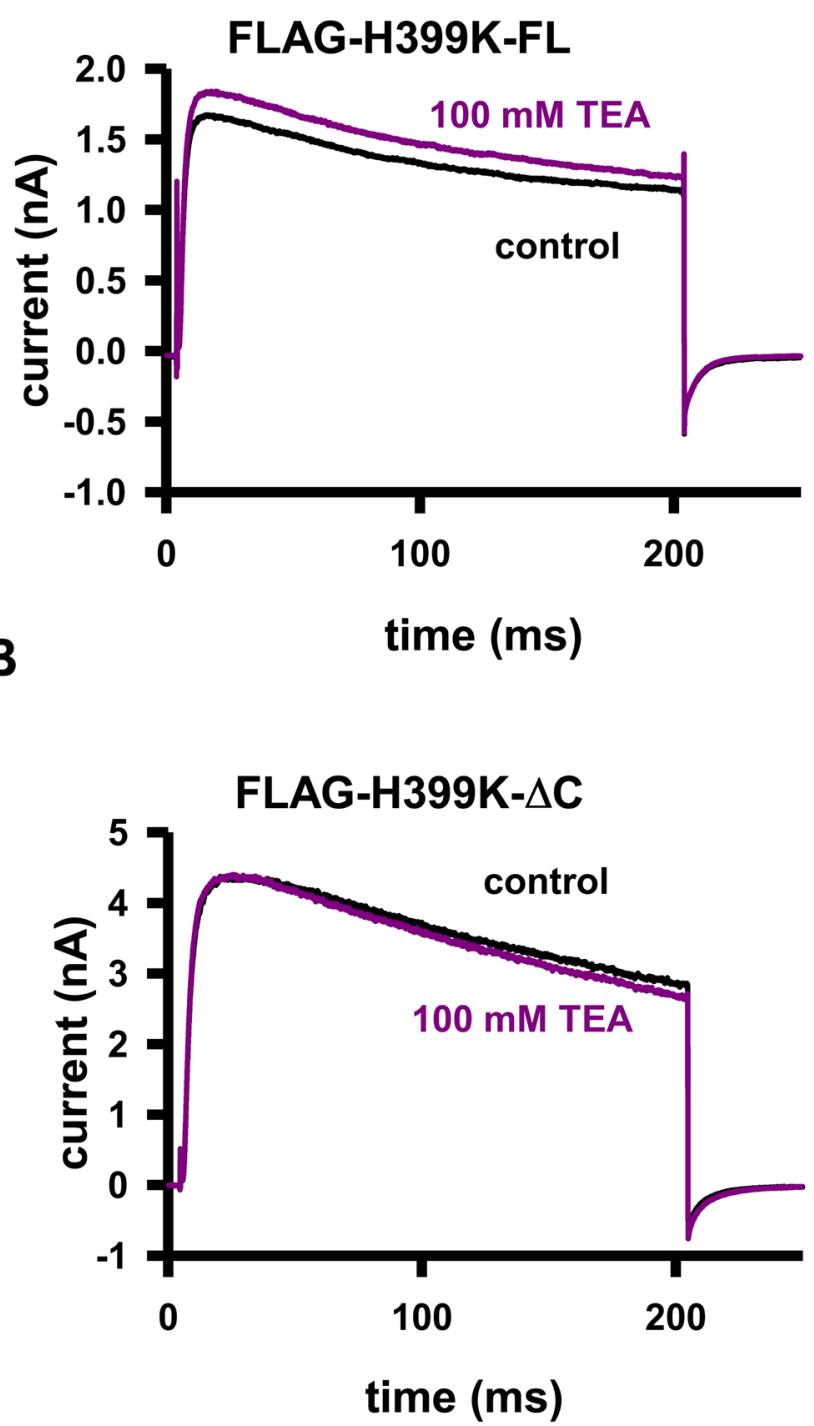

Figure 3. TEA inhibition of full length and C-terminal removed H399K channel. (A) Whole-cell currents of EGFP- and FLAG-tagged H399K-FL channels in CHO cells were recorded during 200-ms-long pulses to $0 \mathrm{mV}$ (not to $+50 \mathrm{mV}$ to reduce whole-cell current) from a holding potential of $-120 \mathrm{mV}$. Traces are shown before (black) and after addition of $100 \mathrm{mM}$ TEA (purple). (B) Currents of the EGFP- and FLAG-tagged H399K- $\Delta \mathrm{C}$ construct in the presence (purple) and absence (black) of $100 \mathrm{mM}$ TEA. The same voltage-clamp protocol was used as on panel A was used to evoke the currents.

A413V-NOHRET, H399K-NOHRET) were expressed either in HEK or CHO. This might mean impaired trafficking and/or conductivity/functionality of these latter constructs.

NOHRET constructs can also target to the cell membrane. Next, we addressed if C-terminal truncated constructs that also lack the "HRET" motif can be expressed in the cell membrane. The Kv1.3 subunits were conjugated to EGFP on the intracellular N-terminus and the extracellular FLAG epitope was cloned into each plasmid encoding point and deletion mutants of the Kv1.3 channel (FLAG-H399K-FL, FLAG-H399K- $\Delta$ C, FLAG-H399K-NOHRET and FLAG-A413V-FL, FLAG-A413V- $\triangle$ C, FLAG-A413V-NOHRET constructs). The distribution of the channel subunits was detected by means of confocal fluorescence microscopy. Figure 4A shows that FLAG-tagged full-length, H399K and A413V mutant Kv1.3 channels were stained with anti-FLAG antibody in both CHO and HEK cells (only CHO is shown, for HEK see Suppl. Figure 5). The same was observed for both $\mathrm{Kv} 1.3$ point mutants that lacked either the complete $\mathrm{C}$-terminus $(\Delta \mathrm{C}$, Fig. $4 \mathrm{~B})$ or the $\mathrm{C}$-terminus plus the "HRET" motif (NOHRET, Fig. 4C). This confirms the presence of all of these constructs in the plasma membrane, though the intracellular retention of truncated channels was elevated as compared to the full-length counterpart as shown by the quantitative analysis of the images (Suppl. Figure 6). Moreover, no difference in membrane 
EGFP-Kv1.3 FLAG-A647 Merge Brightfield

A
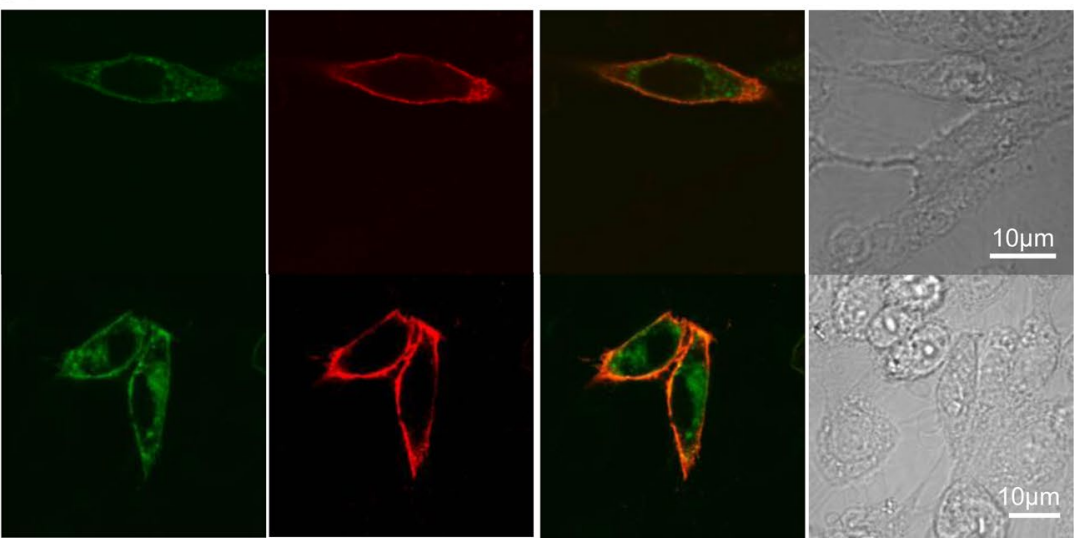

FLAG-A413V-FL

B
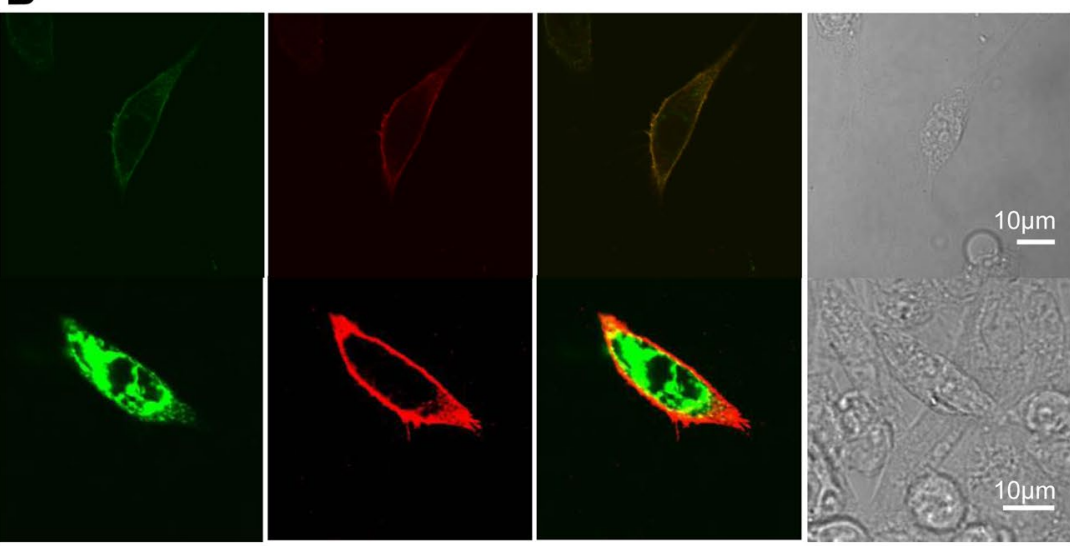

FLAG-A413V- $\Delta$ C

C
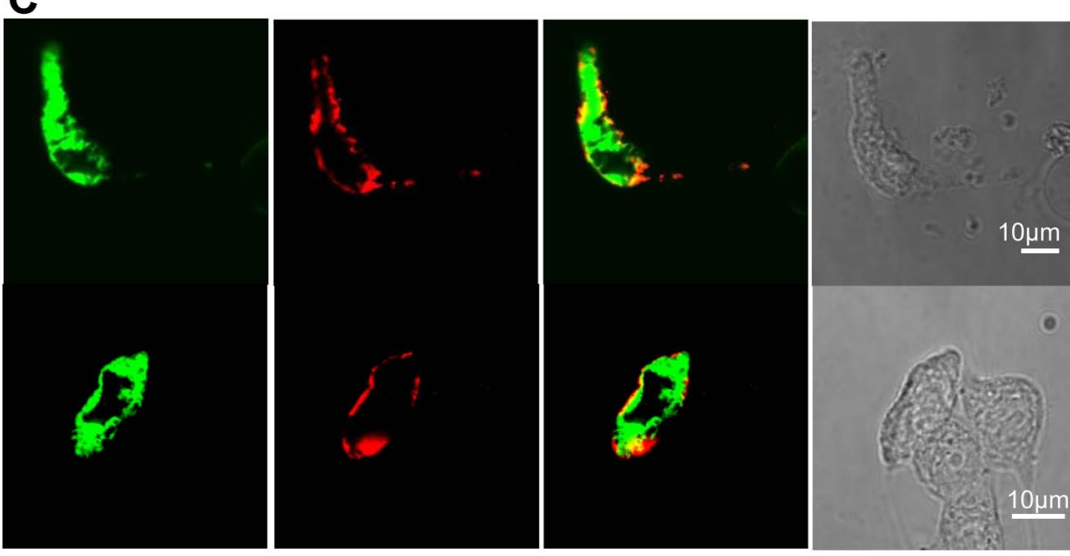

FLAG-H399K-FL

FLAG-H399K- $\Delta$ C

Figure 4. Targeting of deletion mutants of Kv1.3 to the plasma membrane. Representative images of EGFP- and FLAG-tagged Kv1.3 mutant channels expressed in CHO. (A) CHO cells expressing the A413V-FL/H399K-FL, (B) A413V/H399K C-terminal truncated Kv1.3 (A413V- $\Delta$ C/H399K- $\Delta$ C) or C) A413V-NOHRET/H399KNOHRET plasmid is displayed. The $1^{\text {st }}$ column shows the EGFP signal of Kv1.3 channel in green. Cells were labeled with anti-FLAG primary and Alexa Fluor 647 GAMIG secondary antibodies to verify cell membrane localization of the channel protein $\left(2^{\text {nd }}\right.$ column, red). The $3^{\text {rd }}$ column shows the merge of red and green channels of the EGFP and anti-FLAG fluorescence signal whereas brightfield images of the cells are shown in the $4^{\text {th }}$ column. (For details of fluorescent labeling see Materials and Methods).

expression between the $\Delta \mathrm{C}$ and the NOHRET constructs was detected for both A413V and H399K (Suppl. Figure 6). All these data support the hypothesis that Kv1.3 channels can reach the membrane even without the "HRET" motif and instead the gating/conductance of the channel is impaired. 


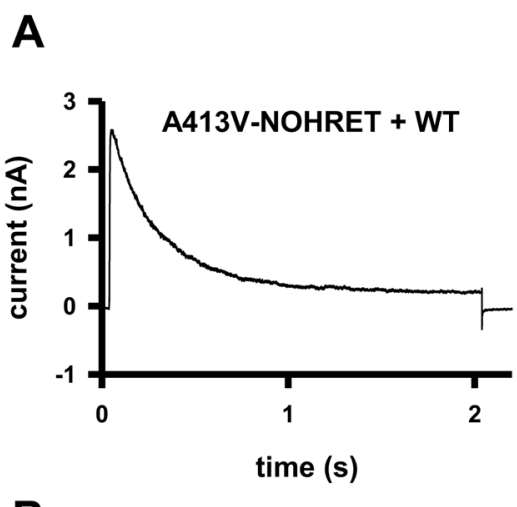

B

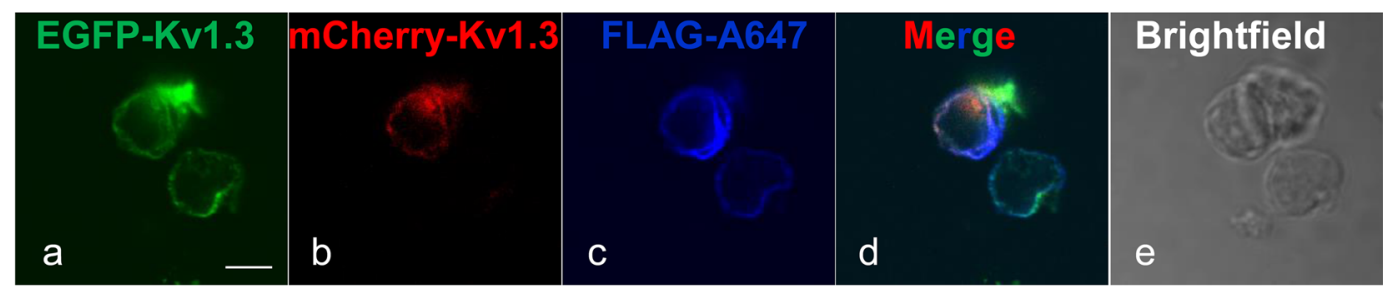

Figure 5. WT Kv1.3 fails to rescue the A413V phenotype when co-transfected with A413V-NOHRET subunits. (A) Whole-cell current recorded in a CHO cell co-transfected with EGFP-tagged A413V-NOHRET and mCherry tagged WT Kv1.3 plasmids (1:1 molar ratio). The cell was depolarized to $+50 \mathrm{mV}$ from a holding potential of $-120 \mathrm{mV}$. (B) Confocal images of a CHO cell expressing both FLAG and EGFP-tagged A413V-NOHRET and mCherry-tagged WT Kv1.3. Cells were stained with anti-FLAG and Alexa-647 GAMIG antibodies. $a$ : A413V-NOHRET (green), $b$ : mCherry-WT Kv1.3 (red), $c$ : FLAG (blue), $d$ : overlay of red, green blue channels, $e$ : brightfield image of the cells. Scale bar is $5 \mu \mathrm{m}$.

Co-expression of wild-type and A413V-NOHRET subunits results in pure wild-type Kv1.3 current. We co-transfected mCherry-tagged full-length, wild-type Kv1.3 channels along with EGFP-A413V-NOHRET plasmid mutants at 1:1 ratio and studied if the presence of the WT subunits can rescue conduction of the channels containing A413V-NOHRET subunits. Assuming that tetrameric channels assemble randomly from individual subunits a $1: 1$ co-transfection ratio should result in predominantly $(87.5 \%)$ heteromeric channels having intermediate inactivation kinetics between $\mathrm{WT}$ and $\mathrm{A} 413 \mathrm{~V}^{22}$. On the contrary, we found that a CHO cell expressing both A413V-NOHRET and WT-Kv1.3 exhibits currents resembling the pure WT current (Fig. 5A); and not the "mixture" of multiple heteromeric currents characterized with various inactivation time constants (also see current trace of A413-FL and WT Kv1.3 in Fig. 1C and Suppl. Figure 1B). The current density of only WT-Kv1.3 channel expressing cells $(3390 \pm 1110 \mathrm{pA} / \mathrm{pF})$ was far higher than those transfected with both WT-Kv1.3 and A413V-NOHRET constructs $(1010 \pm 311 \mathrm{pA} / \mathrm{pF}, \mathrm{p}=0.028)$. Moreover, we transfected CHO cells with a mixture of FLAG-tagged A413V-NOHRET (EGFP-conjugated) and WT-Kv1.3 (mCherry conjugated) and subjected them to anti-FLAG labeling as described in Fig. 4. As displayed in Fig. 5B the FLAG-epitope bearing A413V-NOHRET subunits are labelled by the anti-FLAG antibody, which indicates their membrane targeting.

Gating charge movement of NOHRET channels is absent. To disclose if the conducting pathway or the activation gating is destroyed upon HRET removal in the NOHRET Kv1.3 we assessed the gating properties of WT-NOHRET construct expressed in CHO cells (see Fig. 1B). As a positive control, we expressed the WT-W384F channel, which is a non-conducting mutant of Kv1.3 (homologous to the non-conducting W434F mutant of the Shaker channel ${ }^{32-37}$ ). Figure 6A displays the gating currents recorded in a CHO cells stably expressing Kv1.3-W384F (we recorded gating currents in all 11 cells). The representative $\mathrm{Q}_{\text {on }}-\mathrm{V}$ curve for this cell in the Fig. $6 \mathrm{~B}$ illustrates the sigmoid shape of membrane potential dependence of the integrated gating current, which is a hallmark of voltage-gated ion channels and point out the functionality of the voltage-sensor. When we measured the gating current in cells expressing WT-NOHRET channels no gating current was detected $(n=9$, Fig. $6 \mathrm{~B}$ and $\mathrm{C}$ ) or a miniature gating current was detected at very depolarized test potentials of $+50 \mathrm{mV}$ or higher $(\mathrm{n}=2$, not shown). These indicate that voltage-sensor movement of the channel is compromised when HRET is not present.

Deletion and substitution of the HRET(E) sequence does not affect the function of Kv1.3. Motivated by these findings, mainly those for gating properties of NOHRET constructs, the following mutations were introduced into EGFP-tagged WT Kv1.3: 1) the extended HRET motif, HRET(E) region of the C-tail was deleted only (WT-XHRETE construct); 2) HRET(E) was replaced with a run of five alanines (WT-polyA channel) (see Fig. 1B, bottom sequences) and 3) the HRETE sequence was replaced with 5 alanines in the WT- $\triangle \mathrm{C}$ (WT-Atail channel). After transfecting these mutants and the WT full-length in CHOs we analyzed basic biophysical features for 

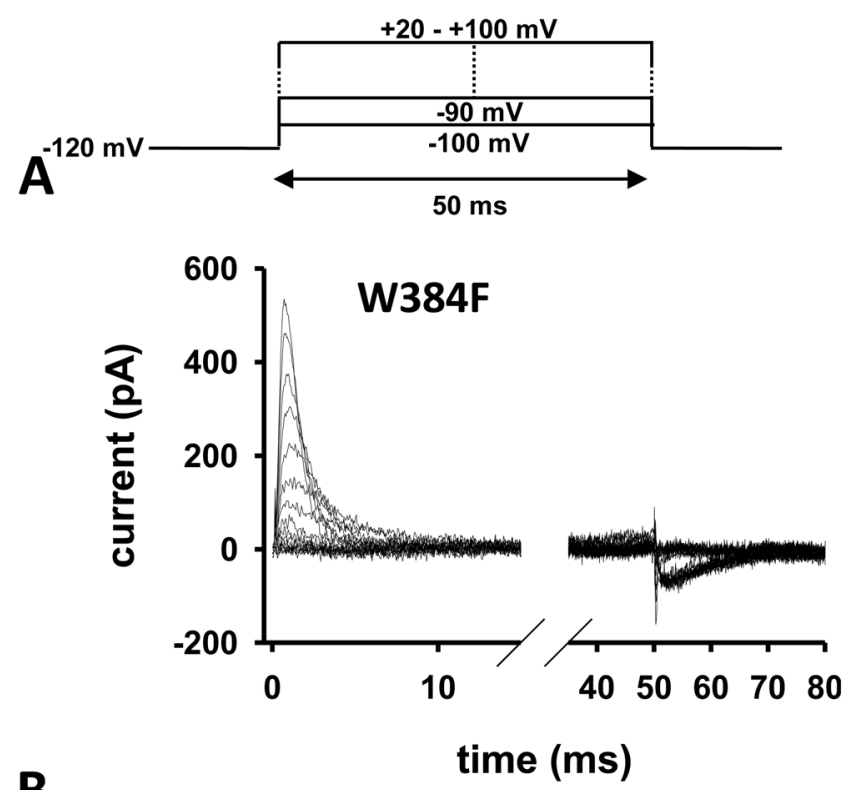

B

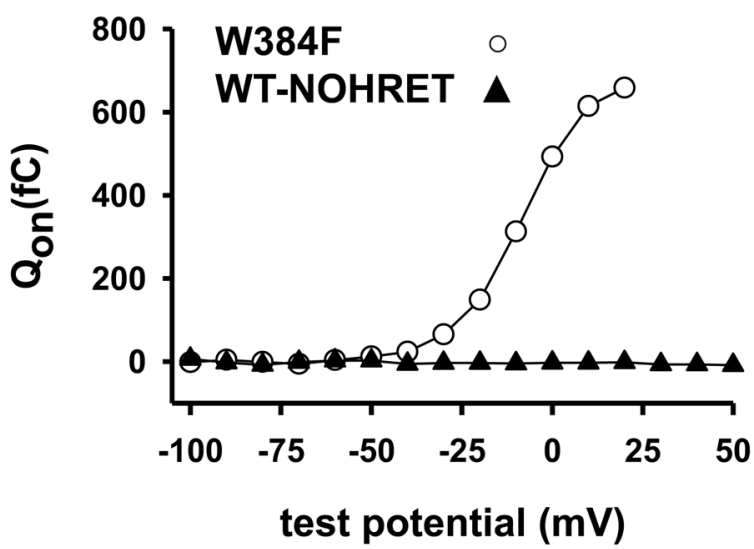

C

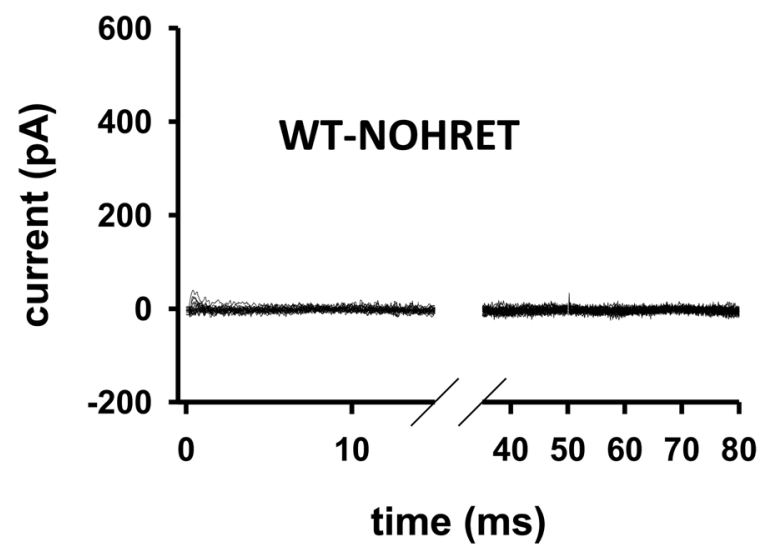

Figure 6. The gating current of WT-NOHRET channels is missing. (A) Gating currents in a WT-W384F channel expressing CHO cell. Cell was held at $-120 \mathrm{mV}$ then depolarized to different test potentials (from $-100 \mathrm{mV}$ to $20 / 100 \mathrm{mV}$ in $10 \mathrm{mV}$ steps) for $50 \mathrm{~ms}$. (B) The test potential dependence of the integrated gating current $\left(\mathrm{Q}_{\mathrm{on}}\right.$, on-gating) for the cells recorded in panel $\mathrm{A}(\mathrm{W} 384 \mathrm{~F})$ and $\mathrm{C}(\mathrm{WT}-\mathrm{NOHRET})$. $\mathrm{Q}_{\text {on }}$ was evaluated as described in the Material and Methods section. (C) Gating current recordings in a CHO cell transfected with WT-NOHRET construct. An identical pulse protocol was applied as in A.

all channel types in outside-out patch configuration. As shown in Fig. 7, all three HRETE-manipulated subunits formed functional and conducting tetramers in the $\mathrm{CHO}$ cell membrane, and no major differences in the kinetic and equilibrium parameters of the gating were found. The activation kinetics was a bit slower for the 


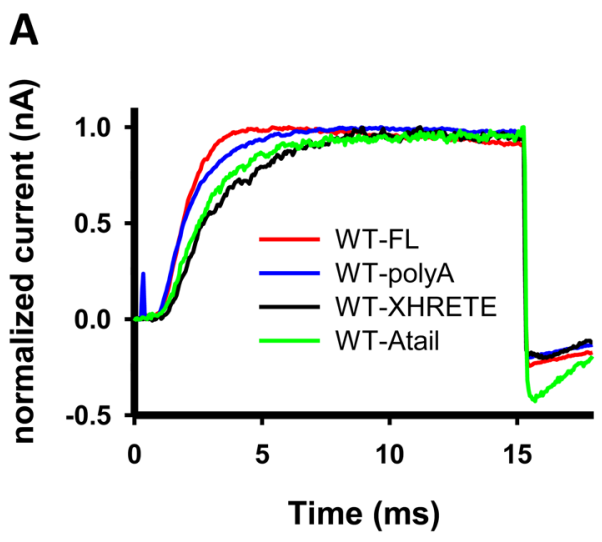

B

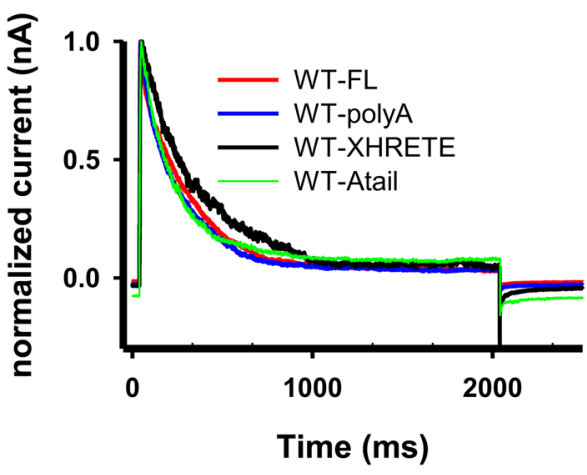

C

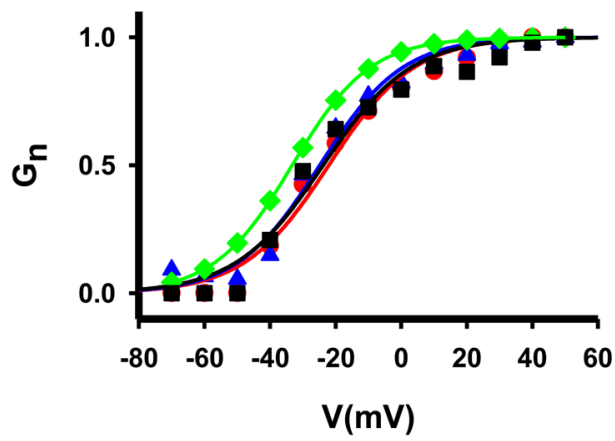

D

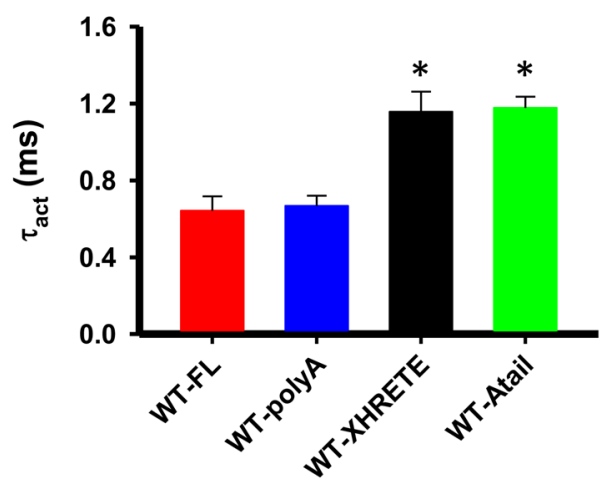

$\mathbf{E}$

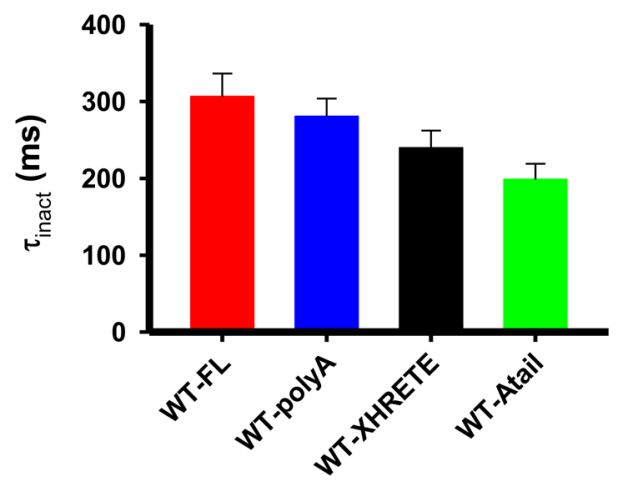

$\mathbf{F}$

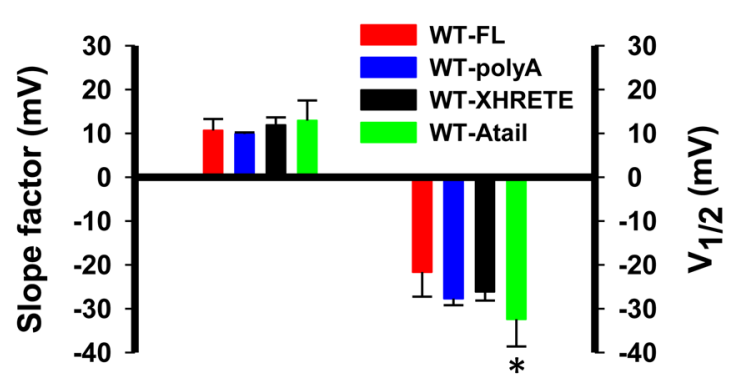

Figure 7. Elimination of the HRETE motif does not affect Kv1.3 operation. (A) Current traces of WT-FL, WTXHRETE, WT-polyA and WT-Atail channels were recorded upon 15-ms-long $+50 \mathrm{mV}$ depolarization to study activation kinetics of the four constructs (red line: WT-FL, blue: WT-polyA, black: WT-XHRETE, WT-Atail: green). For fitting and evaluation of activation kinetics see Materials and Methods. Holding potential was set to $-120 \mathrm{mV}$, measurements were performed in outside-out configuration and currents were normalized to the peak value for the panel A and B. (B) Typical current recordings showing the inactivation kinetics of the WT-FL, WT-polyA, WT-XHRETE and WT-Atail channels. Cells were depolarized to $+40 \mathrm{mV}$ for $2 \mathrm{~s}$ from $-120 \mathrm{mV}$ holding potential, inactivation kinetics was evaluated as described in Materials and Methods. (C) Voltage-dependence of steady-state activation of four Kv1.3 channel phenotypes: the normalized conductancetest potential relationships were obtained and analyzed as described in the Materials and Methods. Normalized conductance as a function of the test potential is shown for a WT-FL (filled circle, red line), a WT-polyA (empty triangles, blue line), a WT-XHRETE (empty square, black line) and WT-Atail (green diamonds, green line) channel expressing cell along with the best fit Boltzmann function. (D-E) The activation and inactivation time constants for the WT-FL, WT-polyA, WT-XHRETE and WT-Atail channels (mean \pm SEM, $\mathrm{n} \geq 6$ ). Asterisk represents significant difference, $\mathrm{p}=0.05$. (F) Parameters of steady-state activation (slope factor $(k)$, left and midpoint $\left(V_{1 / 2}\right)$, right) are displayed for the four constructs (mean \pm SEM, $\mathrm{n} \geq 6$ ). 
WT-XHRETE and WT-Atail constructs as compared to WT-FL ( $<<0.001$ for both), but not the inactivation kinetics $(p=0.13)$. The half maximal voltage $\left(V_{1 / 2}\right)$ of the steady-state activation was only different for WT-Atail channels $(p=0.005$, leftward shift) but the slope factor $(k)$ was the same for all HRETE-mutants (WT-polyA, WT-Atail and WT-XHRETE, $\mathrm{p}=0.284$ ). Furthermore, we assessed the single-channel conductance for all four phenotypes and obtained that removal/replacement of HRETE-motif in Kv1.3 channels did not influence the unitary conductance (Suppl. Figure 7, p =0.085). Moreover, FLAG-epitope bearing WT-Atail channels could be detected on the cell surface (Suppl. Figure 8). All these data clearly demonstrate that the HRETE motif is not vital for the operation of Kv1.3: the channel is present in the plasma membrane, and it is functional in the absence of this motif.

\section{Discussion}

Membrane targeting of $\mathrm{Kv}$ channels has been studied by multiple groups, as it is a key player in the regulation of various cellular processes including action potential regulation or immune response ${ }^{38}$. To draw appropriate conclusion for trafficking it is critically important to demonstrate unambiguously that the current recorded is a consequence of the ion channel genes transfected into the cells and not influenced by endogenous $\mathrm{K}^{+}$channel subunits in the expression system. To do this we used kinetically or pharmacologically tagged Kv1.3 subunits with properties that uniquely distinguish homotetrameric channels formed by transfected subunits from endogenous $\mathrm{K}^{+}$channels or hetermultimers of endogenous and transfected channel subunits. If the A413V- $\Delta \mathrm{C}$ subunits reach the plasma membrane because they form heterotetramers with endogenous WT Kv1.3 in HEK cells, then the inactivation kinetics of the whole-cell current should be fitted with sum of multiple exponential decay functions (see Suppl. Figure 1). On the contrary, we obtained that $\tau_{i}$ for A413V-FL $(4.1 \pm 0.6 \mathrm{~ms})$ was the same as that of the $\mathrm{A} 413 \mathrm{~V}-\Delta \mathrm{C}(5.7 \pm 0.5 \mathrm{~ms}, \mathrm{p}=0.15)$, and the decaying part of the curves could be fitted with a single-exponential function (data not shown). The expression of A413V- $\Delta \mathrm{C}$ current in $\mathrm{CHO}$ cells, which do not exhibit any voltage-gated $\mathrm{K}^{+}$conductance, supports the scenario as well that $\Delta \mathrm{C}$-truncated mutants are targeted to the plasma membrane without their combination with full-length Kv1.x subunits.

The outcomes with the $\mathrm{H} 399 \mathrm{~K}$ mutant also support the hypothesis that C-terminus removal downstream of the "HRET" region does not prevent targeting of Kv1.3 to the cell membrane. For H399K- $\triangle \mathrm{C}$ and H399K-FL the inhibition by $100 \mathrm{mM}$ TEA was negligible, which verifies that there was no mixing of $\mathrm{H} 399 \mathrm{~K}-\Delta \mathrm{C}$ and WT Kv1.3 subunits in the ER (we used CHO cells here as well, which have no Kv1 subunits), otherwise we should have seen block by TEA ${ }^{27,31}$. In addition, we detected the FLAG epitopes of both A413V- $\Delta \mathrm{C}$ and H399K- $\Delta \mathrm{C}$ subunits in transfected cells (CHO and HEK) with immunocytochemistry in non-permeabilized cells, which serves as an additional proof for their plasma membrane localization (Fig. 4 and Suppl. Figure 5) Interestingly, we also found that A413V-NOHRET or H399K-NOHRET bearing the FLAG-epitope showed a membrane signal upon anti-FLAG staining, although patch-clamp experiments revealed that these deletion mutants produced no current.

Our results somewhat contradict the findings of previous studies which emphasized the importance of the C-terminal region downstream of the "HRET" motif in Kv1.3, Kv1.2 and Kv1.1 in regulating trafficking ${ }^{14,15}$. The importance of a diacidic signal (E483/484) in the C-terminal region of Kv1.3 was suggested to control surface expression via interaction with Sec24 (a coat protein of vesicular transport) ${ }^{14}$. Another study demonstrated that alanine-scanning mutational analysis of glutamates at residues 438/440/442 (in our construct) slightly increased ER retention only for triple mutants, and Sec24a and Sec24b knock-down inhibited from-ER-to-Golgi trans$\operatorname{port}^{15}$. Furthermore, the removal of a much shorter fraction of the C-terminus, as compared to our study, was sufficient to cease the anterograde transport of Kv1.3 ${ }^{14}$. On the contrary, Kv1.3 in our hands is targeted to the plasma membrane in the absence of the entire C-terminus that includes the HRET sequence, however, increase in the intracellular retention of the channel protein was observed in the truncated channels. (Suppl. Figure 6, Fig. 4). We suppose that the use of different Kv1.3 genes (human in this study vs rat and mouse in others) cannot explain the difference due to multiple reasons: 1) we have reported previously that various C-truncated rat Kv1.3 channels had high membrane expression ${ }^{16}$, 2) the mouse, rat and human Kv1.3 genes have high homology, 3) in our recent paper we showed that the C-terminal deletion of WT hKv1.3 (stop codon after HRET motif) results in the expression of currents comparable to that of the full-length WT Kv1.3 ${ }^{10}$. We think that the lack of or the very low expression detected by others for the C-terminal truncated constructs can be attributed to the cell lines used to express these constructs ${ }^{12,14,29}$. For example, glycosylation or other post-translational modifications can modify the surface expression level of Kv1.x channels; or the heteromerization of other Kv subunits that are endogenously present in these cells with Kv1.3 may prevent the trafficking to the plasma membrane ${ }^{29,39-45}$. Alternatively, the mutations cited above may create a retention signal that affect the trafficking of the channels to the membrane. As shown here, we ruled out cell line specific conclusions by using two cell lines and mutation specific effects by using two point mutants having unique characteristics.

On the other hand, our results are in harmony with others showing that the lack or modification of the HRET sequence does not terminate cell surface expression of Kv1.1 or the Shaker channel without the equivalent "HRE" sequence makes it to the membrane, similar to Kv1.3 in this study ${ }^{12,13}$. In addition to trafficking, the role of the HRET(E) sequence was suggested in regulating the conductance of various channels. For example, the arginine in the HRET(E) sequence of Kv1.1 is critical to form a conductive channel and the Shaker channel lacking the HRE sequence shows altered steady-state activation gating ${ }^{12,13}$. Our results partially agree with these studies: the C-terminally truncated Kv1.3 that lacks the HRET sequence is expressed in the surface membrane but does not conduct $\mathrm{K}^{+}$current.

However, we went much further in understanding this phenomenon. First, WT Kv1.3 could not rescue the conductance of the A413V-NOHRET subunits in CHO cells, i.e., co-transfection of these two subunits resulted in pure homotetrameric WT currents. Fluorescence signals clearly showed the presence of both subunits in the plasma membrane, thus, interpretation of these results is that either WT Kv1.3 and A413V-NOHRET subunits 
do not form heterotetramers or that the presence of A413V-NOHRET subunits in a heteroteramer renders the channel non-conductive in a dominant negative manner. The fact that cells transfected with WT-Kv1.3 had much greater current density as compared to cells expressing both constructs support the formation of non-conducting heterotetramers in the cell membrane (i.e., some WT subunits are engaged in non-conducting heterotetramers). As tetramerization of Kv1.3 is governed by the N-terminal tetramerization domain, rather than the C-terminus, we favor the dominant-negative effect of the mutant subunits (as has been demonstrated for other mutations as well $)^{1,46-48}$.

The HRET(E) is sequence is located in the part of the C-terminal that is proximal to the activation gate or may be part of it ${ }^{49}$. Thus, mutation in this region may profoundly alter activation gating of the channels, the coupling of the voltage-sensor movement to the activation gate or both while leaving the voltage-sensor movement intact. To test this assumption, we determined the gating currents of WT-NOHRET channels. To our surprise, we found that majority of the cells expressing this channel did not exhibit gating currents that resemble the gating current of the non-conducting W384F mutant (Fig. 6), which was used as a control. As fluorescence signals confirm the surface expression of the NOHRET construct this result suggests that voltage-sensor movement is impaired in the NOHRET Kv1.3 that lacks the full C-terminus including the HRET sequence. The origin of this unknown "reverse coupling" (i.e. movement of the voltage sensor is impaired by modification of the activation gate region) is unknown, but it does not seem to be specific for the HRET sequence. When we deleted just the HRETE sequence (WT-XHRETE construct) or substituted with alanines (WT-polyA) and left the rest of the carboxyl-terminus intact, or changed HRETE for five alanines in the WT-Atail construct (and rest of $\mathrm{C}$-terminus was removed) the conductance of Kv1.3 was recovered. So it seems that the lack of a peptide strand on the $\mathrm{C}$-terminus at the activation gate renders the channels non-conductive and any replacement may substitute for the HRETE sequence, at least in Kv1.3.

In summary, we demonstrated that Kv1.3 channel trafficking to the plasma membrane is preserved even if the whole C-terminus, including the HRET sequence is deleted. This finding highlights that trafficking motifs may not be universal and their importance must be tested for each channel/expression system combination. A similar conclusion can be drawn for the role of the HRET sequence in regulating channel conductance ${ }^{12,49}$ : in case of Kv1.3 even an alanine substitution of the HRETE sequence restored channel function. Based on this and other recent papers the presence of the $\mathrm{C}$-terminal amino acids adjacent to the activation gate in $\mathrm{Kv} 1.3$ are important for maintaining ion conductance, whereas distant $\mathrm{C}$-terminal amino acids govern interactions with other proteins or confer cholesterol sensitivity to the Kv1.3 $3^{50}$.

\section{Materials and Methods}

Kv1.3 channel constructs. The wild-type human Kv1.3 channel (shorter version of Kv1.3, composed of 523 amino acids acc. \#: M8521751) was subcloned into pEGFP-C1 plasmid (Clontech, USA) using Bgl II and EcoR I restriction sites (Thermofisher, Hungary). For sticky-end ligation, T4 DNA ligase (Thermofisher, Hungary) was used according to the standard protocol. The deletion ( $\triangle \mathrm{C}$, NOHRET, XHRETE) and insertion/point (FLAG tag, polyA, Atail, W384F) mutants were generated with flanking-primer mutagenesis method. Point-mutant (H399K, A413V), full-length constructs in pRc-CMV were made before as described in ${ }^{22,25}$. For co-transfection of wild type (WT) and mutant Kv1.3 plasmids mCherry tagged WT Kv1.3 was generated in two steps with PCR-based cloning: first EGFP was replaced with mCherry in pEGFP-C1 plasmid, then the wild-type Kv1.3 gene was subcloned into this pmCherry-C1 plasmid with EcoR I and Bgl II enzymes. All constructs were sequenced at the Clinical Genomics Center at University of Debrecen.

Cell culture and transfection. HEK-derived tsA-201 (later we call them HEK for simplicity) and CHO (chinese hamster ovary, both from ATCC, Germany) cells were cultured in DMEM medium (Sigma-Aldrich Ltd., Hungary), which contained 10\% FBS, $1 \mathrm{mM}$ Na-pyruvate, and 200 units penicillin/streptomycin. Cells were maintained at $37^{\circ} \mathrm{C}$ in a humidified atmosphere of $5 \%$ of $\mathrm{CO}_{2}$ and $95 \%$ air. Cells were passaged every $2-3$ days. Transfections of DNA plasmids were performed using Lipofectamine $2000^{\mathrm{TM}}$ (Life Technologies, Hungary) according to the manufacturer's protocol. The patch-clamp and immunocytochemistry experiments were performed 24 hours post transfection. The CHO cell line stably expressing EGFP-tagged W384F-Kv1.3 was established as detailed in ref. ${ }^{10}$.

Electrophysiology. HEK and $\mathrm{CHO}$ cells transfected with various $\mathrm{Kv} 1.3$ channel constructs were washed with standard extracellular solution (composition was in mM: $145 \mathrm{NaCl}, 5 \mathrm{KCl}, 1 \mathrm{MgCl}_{2}, 2.5 \mathrm{CaCl}_{2}, 5.5$ glucose, 10 HEPES, pH: 7.35), and plated onto cell culture $35-\mathrm{mm}$ petri dishes. Kv1.3 currents were recorded in whole-cell and outside-out configuration using an Axopatch 200B amplifier (Molecular Devices, Sunnyvale, CA, USA) as previously described[ $]^{52}$. The pipette filling solution consisted of (in $\mathrm{mM}$ ): $140 \mathrm{KF}, 11 \mathrm{~K}_{2} \mathrm{EGTA}, 1 \mathrm{CaCl}_{2}, 2 \mathrm{MgCl}_{2}$, and 10 HEPES ( $\mathrm{pH} 7.20, \sim 295$ mOsm). TEA (tetraethylammonium) containing bath solution contained (in mM): 100 TEA-Cl, $45 \mathrm{NaCl}, 5 \mathrm{KCl}, 1 \mathrm{MgCl}_{2}, 2.5 \mathrm{CaCl}_{2}, 5.5$ glucose, 10 HEPES (pH 7.35, $305 \mathrm{mOsm}$ ). For gating-current and single-channel measurement the external bath did not contain $\mathrm{Na}^{+}$and the composition was the following (in mM): 145 choline-Cl, $5 \mathrm{KCl}, 1 \mathrm{MgCl}_{2}, 2.5 \mathrm{CaCl}_{2}, 5.5$ glucose, $10 \mathrm{HEPES}$ (pH 7.35, $\left.305 \mathrm{mOsm}\right)\left(\mathrm{Na}^{+}\right.$had to be omitted from the solution since some $\mathrm{CHO}$ cells expressed inward $\mathrm{Na}^{+}$current). $\mathrm{P} / 5$ protocol for online leak subtraction was applied to minimize capacitance and leak errors during the measurements with the A413V mutant. Perfusion of the cell with different test solutions was achieved using a gravity-flow perfusion setup, and excess fluid was removed continuously.

The current density was defined as the ratio of peak current detected at $+50 \mathrm{mV}$ test potential and the whole-cell capacitance (read from the compensatory circuit of the amplifier). The remaining fraction of the 
current (RF) for TEA inhibition was defined as the ratio of the peak current measured after and before perfusion with $100 \mathrm{mM}$ TEA.

The activation kinetics of the current was characterized by fitting the Hodgkin-Huxley $(\mathrm{HH})$ model $\left(\mathrm{I}(\mathrm{t})=\mathrm{I}_{\mathrm{a}}\right.$ $\times\left(1-\exp \left(-t / \tau_{a}\right)\right)^{4}+C$ where $I_{a}$ is the amplitude of the activating current component; $\tau_{a}$ is the activation time constant of the current; $\mathrm{C}$ : constant) to the rising phase of the current traces evoked by 15-ms-long depolarizations to $+50 \mathrm{mV}$. The activation time constant characteristic of a given cell was determined as the average of the time constants obtained upon three sequential depolarizations repeated every $15 \mathrm{~s} . \mathrm{P} / 5$ protocol for online leak subtraction was applied.

The inactivation kinetics of the current was characterized by fitting a single exponential function $\left(\mathrm{I}(\mathrm{t})=\mathrm{I}_{0}\right.$ $\times \exp \left(-\mathrm{t} / \tau_{\text {in }}\right)+\mathrm{C}, \mathrm{I}_{0}$ : amplitude of current, $\tau_{\text {in }}$ : inactivation time constant, C: steady-state value of whole-cell current at the end of the pulse) to the decaying part of the current traces evoked by 2 -s-long depolarizations to $+40 \mathrm{mV}$ from a holding potential of $-120 \mathrm{mV}$. The inactivation time constant for a given cell was determined as for $\tau_{\mathrm{a}}$, except pulses were delivered every $60 \mathrm{~s}$.

The voltage-dependence of steady-state activation relationships were obtained as follows. The cells were held at $-120 \mathrm{mV}$ holding potential and depolarized to various test potentials ranging from -70 up to $+50 \mathrm{mV}$ in $10 \mathrm{mV}$ steps at every $30 \mathrm{~s}$. Peak whole-cell conductance $(\mathrm{G}(V))$ at each test potential was calculated from the peak current $\left(\mathrm{I}_{\mathrm{p}}\right)$ at test potential $V$ and the $\mathrm{K}^{+}$reversal potential $\left(\mathrm{E}_{\mathrm{r}}=-85 \mathrm{mV}\right)$ using $\mathrm{G}(V)=\mathrm{I}_{\mathrm{p}} /\left(V-\mathrm{E}_{\mathrm{r}}\right)$. The $G(V)$ values were normalized for the maximum conductance and plotted as a function of test potential and the Boltzmann-function was fitted to the data points: $\mathrm{G}_{\mathrm{N}}=1 /\left(1+\exp \left[-\left(V-V_{1 / 2}\right) / k\right]\right.$, where $\mathrm{G}_{\mathrm{N}}$ is the normalized conductance, $V$ is the test potential, $V_{1 / 2}$ is the midpoint and $k$ is the slope of the function.

Gating currents were determined using a non-conducting Kv1.3 mutant (W384F-Kv1.3). A voltage step protocol was applied from $-100 \mathrm{mV}$ up to $20-100 \mathrm{mV}$ with an increment of $10 \mathrm{mV}$, each step lasted for $50 \mathrm{~ms}, \mathrm{P} / 5$ protocol was used for leak subtraction (pulses were opposite to the test potential) to reduce capacitance and leak errors during the measurements. The gating charge was calculated upon the integration of the area under gating current traces.

Immunocytochemistry. For Fig. 5 and Suppl. Figure 5: HEK and CHO cells expressing FLAG bearing EGFP-Kv1.3 plasmids were plated onto poly-L-lysine coverslips and incubated for 1 hour $\left(37^{\circ} \mathrm{C}\right.$, humidified, $\left.5 \% \mathrm{CO}_{2}\right)$. Then cells were fixed with $1 \%$ formaldehyde and labeled with mouse anti-FLAG M2 antibody (1:1000, Sigma-Aldrich Ltd., Hungary). Secondary antibodies (goat anti-mouse with Alexa647, Thermofisher, Hungary) were added to the cells for 1 hour. Finally, coverslips were mounted onto slides with Fluoromount G (eBioScience, USA). Zeiss LSM 510 META and Olympus FV-1000 microscopes were used to take confocal images of the cells. A He-Ne laser was selected to excite Alexa647 and mCherry (line 633 and $543 \mathrm{~nm}$ ) and an Argon laser (line $488 \mathrm{~nm}$ ) to visualize EGFP. The thickness of the slices was set to $1 \mu \mathrm{m}$.

For Fig. 4. and Suppl. Figure 8: CHO cells were plated into 8-well chamber and transfected with FLAG bearing EGFP-Kv1.3 plasmids $\left(37^{\circ} \mathrm{C}\right.$, humidified, $\left.5 \% \mathrm{CO}_{2}\right)$. After 24 hours the cells were washed and then fixed with $1 \%$ formaldehyde and labeled with mouse anti-FLAG M2 antibody for 2 hours, $37^{\circ} \mathrm{C}(1: 500$, Sigma-Aldrich Ltd., Hungary). Secondary antibodies (goat anti-mouse with Alexa647, 1:500, Thermofisher, Hungary) were added to the cells for 1 hour. Zeiss LSM 510 META and Olympus FV-1000 microscopes were used to take confocal images of the cells. A He-Ne laser was selected to excite Alexa647 (line $633 \mathrm{~nm}$ ) and an Argon laser (line $488 \mathrm{~nm}$ ) to visualize EGFP. The thickness of the slices was set to $1 \mu \mathrm{m}$.

Western blotting. Protein samples were separated by SDS-PAGE and transferred to PVDF membranes (Millipore, Billerica, MA, USA) after electrophoresis. The membranes were blocked with milk powder, and immunoblotted with mouse-anti-FLAG M2 (Sigma-Aldrich Ltd., Hungary) or rabbit-anti-actin (Sigma-Aldrich Ltd., Hungary) primary and anti-mouse IgG HRP-linked or anti-rabbit IgG HRP-linked secondary antibodies (Cell Signaling Technology, Inc., Beverly, MA), respectively. Blots were developed with ECL reagent (Thermo Scientific Inc., Vantaa, Finland). The blots were then visualized with the FluorChem Q MultiImage III Western blot imaging system (ProteinSimple, USA).

Statistical analysis. Data are reported as the mean \pm standard error. Means were compared using Student's t-test or one-way ANOVA. P-value was set to 0.05 . Statistical analyses were performed using SigmaPlot version 10.0 (Systat Software Inc.).

Reagents. All reagents were purchased from Sigma-Aldrich (St. Louis, MO, USA), unless stated otherwise.

Data availability statement. Data published in this study available upon reasonable request from the corresponding author.

\section{References}

1. Deutsch, C. The birth of a channel. Neuron 40, 265-276 (2003).

2. Deutsch, C. Potassium Channel Ontogeny. Annual Review of Physiology 64, 19-46, https://doi.org/10.1146/annurev. physiol.64.081501.155934 (2002).

3. Sewing, S., Roeper, J. \& Pongs, O. Kv beta 1 Subunit Binding Specific for Shaker-Related Potassium Channel Alpha Subunits. Neuron 16, 455-463, https://doi.org/10.1016/s0896-6273(00)80063-x (1996).

4. Liman, E. R., Hess, P., Weaver, F. \& Koren, G. Voltage-sensing residues in the $\mathrm{S} 4$ region of a mammalian K+ channel. Nature 353, 752-756, https://doi.org/10.1038/353752a0 (1991).

5. Lai, H. C. \& Jan, L. Y. The distribution and targeting of neuronal voltage-gated ion channels. Nat Rev Neurosci 7, 548-562 (2006).

6. Burke, N. A. et al. Distinct structural requirements for clustering and immobilization of K+ channels by PSD-95. J Gen Physiol 113, 71-80 (1999).

7. Kim, E. \& Sheng, M. Differential K+ channel clustering activity of PSD-95 and SAP97, two related membrane-associated putative guanylate kinases. Neuropharmacology 35, 993-1000 (1996). 
8. Kim, E., Niethammer, M., Rothschild, A., Jan, Y. N. \& Sheng, M. Clustering of Shaker-type K+ channels by interaction with a family of membrane-associated guanylate kinases. Nature 378, 85-88 (1995).

9. Gong, J. et al. Differential stimulation of PKC phosphorylation of potassium channels by ZIP1 and ZIP2. Science 285, 1565-1569 (1999).

10. Szilágyi, O., Boratkó, A., Panyi, G. \& Hajdu, P. The role of PSD-95 in the rearrangement of Kv1.3 channels to the immunological synapse. Pflugers Arch - Eur J Physiol 465, 1341-1353, https://doi.org/10.1007/s00424-013-1256-6 (2013).

11. Zhu, J., Watanabe, I., Gomez, B. \& Thornhill, W. B. Trafficking of Kv1.4 potassium channels: interdependence of a pore region determinant and a cytoplasmic C-terminal VXXSL determinant in regulating cell-surface trafficking. Biochemical Journal 375, 761-768, https://doi.org/10.1042/bj20030885 (2003).

12. Zhu, J., Gomez, B., Watanabe, I. \& Thornhill, W. B. Kv1 potassium channel C-terminus constant HRETE region: arginine substitution affects surface protein level and conductance level of subfamily members differentially. Molecular Membrane Biology 24, 194-205, https://doi.org/10.1080/09687860601066309 (2007).

13. Lu, Z., Klem, A. M. \& Ramu, Y. Coupling between Voltage Sensors and Activation Gate in Voltage-gated K+ Channels. The Journal of General Physiology 120, 663-676, https://doi.org/10.1085/jgp.20028696 (2002).

14. Martínez-Mármol, R. et al. A non-canonical di-acidic signal at the C-terminus of Kv1.3 determines anterograde trafficking and surface expression. Journal of Cell Science 126, 5681-5691, https://doi.org/10.1242/jcs.134825 (2013).

15. Spear, J. M. et al. Kv1.3 contains an alternative C-terminal ER exit motif and is recruited into COPII vesicles by Sec. 24a. BMC biochemistry 16, 16, https://doi.org/10.1186/s12858-015-0045-6 (2015).

16. Hajdu, P. et al. The C-terminus SH3-binding domain of Kv1.3 is required for the actin-mediated immobilization of the channel via cortactin. Molecular Biology of the Cell 26, 1640-1651, https://doi.org/10.1091/mbc.E14-07-1195 (2015).

17. Herrmann, S., Ninkovic, M., Kohl, T., Lorinczi, E. \& Pardo, L. A. Cortactin controls surface expression of the voltage-gated potassium channel K(V)10.1. J Biol Chem 287, 44151-44163, https://doi.org/10.1074/jbc.M112.372540 (2012).

18. Cheng, L., Yung, A., Covarrubias, M. \& Radice, G. L. Cortactin is required for N-cadherin regulation of Kv1.5 channel function. J Biol Chem 286, 20478-20489, https://doi.org/10.1074/jbc.M111.218560 (2011).

19. Williams, M. R., Markey, J. C., Doczi, M. A. \& Morielli, A. D. An essential role for cortactin in the modulation of the potassium channel Kv1.2. Proc Natl Acad Sci USA 104, 17412-17417, https://doi.org/10.1073/pnas.0703865104 (2007).

20. Jugloff, D. G., Khanna, R., Schlichter, L. C. \& Jones, O. T. Internalization of the Kv1.4 potassium channel is suppressed by clustering interactions with PSD-95. J Biol Chem 275, 1357-1364 (2000).

21. Panyi, G. et al. Colocalization and nonrandom distribution of Kv1.3 potassium channels and CD3 molecules in the plasma membrane of human T lymphocytes. Proc Natl Acad Sci USA 100, 2592-2597, https://doi.org/10.1073/pnas.0438057100 (2003).

22. Panyi, G. \& Deutsch, C. Assembly and suppression of endogenous Kv1.3 channels in human T cells. J Gen Physiol 107, 409-420 (1996).

23. Panyi, G., Sheng, Z. \& Deutsch, C. C-type inactivation of a voltage-gated $\mathrm{K}+$ channel occurs by a cooperative mechanism. Biophys J 69, 896-903, https://doi.org/10.1016/S0006-3495(95)79963-5 (1995).

24. Jiang, B., Sun, X., Cao, K. \& Wang, R. Endogenous Kv channels in human embryonic kidney (HEK-293) cells. Molecular and cellular biochemistry 238, 69-79 (2002).

25. Zhu, G., Zhang, Y., Xu, H. \& Jiang, C. Identification of endogenous outward currents in the human embryonic kidney (HEK 293) cell line. Journal of neuroscience methods 81, 73-83 (1998).

26. Somodi, S. et al. $\mathrm{pH}$-dependent modulation of Kv1.3 inactivation: role of His399. American journal of physiology. Cell physiology 287, C1067-1076, https://doi.org/10.1152/ajpcell.00438.2003 (2004).

27. Heginbotham, L. \& MacKinnon, R. The aromatic binding site for tetraethylammonium ion on potassium channels. Neuron 8 , 483-491 (1992).

28. Yellen, G., Jurman, M. E., Abramson, T. \& MacKinnon, R. Mutations affecting internal TEA blockade identify the probable poreforming region of a K+ channel. Science 251, 939-942 (1991).

29. Bartok, A. et al. Margatoxin is a non-selective inhibitor of human $\mathrm{Kv} 1.3 \mathrm{~K}+$ channels. Toxicon: official journal of the International Society on Toxinology 87, 6-16, https://doi.org/10.1016/j.toxicon.2014.05.002 (2014).

30. Helms, L. M. et al. Margatoxin binds to a homomultimer of $\mathrm{K}(\mathrm{V}) 1.3$ channels in Jurkat cells. Comparison with $\mathrm{K}(\mathrm{V}) 1.3$ expressed in CHO cells. Biochemistry 36, 3737-3744, https://doi.org/10.1021/bi962351p (1997).

31. MacKinnon, R. \& Yellen, G. Mutations affecting TEA blockade and ion permeation in voltage-activated K+ channels. Science 250, 276-279 (1990)

32. Perozo, E., MacKinnon, R., Bezanilla, F. \& Stefani, E. Gating currents from a nonconducting mutant reveal open-closed conformations in Shaker K+ channels. Neuron 11, 353-358 (1993).

33. Yang, Y., Yan, Y. \& Sigworth, F. J. How does the W434F mutation block current in Shaker potassium channels? J Gen Physiol 109, 779-789 (1997).

34. Starkus, J. G., Kuschel, L., Rayner, M. D. \& Heinemann, S. H. Macroscopic Na+ currents in the "Nonconducting" Shaker potassium channel mutant W434F. J Gen Physiol 112, 85-93 (1998).

35. Varga, Z., Rayner, M. D. \& Starkus, J. G. Cations affect the rate of gating charge recovery in wild-type and W434F Shaker channels through a variety of mechanisms. J Gen Physiol 119, 467-485 (2002).

36. Kubota, T., Correa, A. M. \& Bezanilla, F. Mechanism of functional interaction between potassium channel Kv1.3 and sodium channel NavBetal subunit. Scientific reports 7, 45310, https://doi.org/10.1038/srep45310 (2017).

37. Eil, R. et al. Ionic immune suppression within the tumour microenvironment limits T cell effector function. Nature 537, 539-543, https://doi.org/10.1038/nature19364 (2016).

38. Hu, L. et al. Blockade of Kv1.3 Potassium Channels Inhibits Differentiation and Granzyme B Secretion of Human CD8+ T Effector Memory Lymphocytes. PLOS ONE 8, e54267, https://doi.org/10.1371/journal.pone.0054267 (2013).

39. Sutachan, J. J. et al. Effects of Kv1.1 channel glycosylation on C-type inactivation and simulated action potentials. Brain Research 1058, 30-43, https://doi.org/10.1016/j.brainres.2005.07.050 (2005).

40. Watanabe, I. et al. The glycosylation state of Kv1.2 potassium channels affects trafficking, gating, and simulated action potentials. Brain Research 1144, 1-18, https://doi.org/10.1016/j.brainres.2007.01.092 (2007).

41. Watanabe, I., Zhu, J., Recio-Pinto, E. \& Thornhill, W. B. Glycosylation Affects the Protein Stability and Cell Surface Expression of Kv1.4 but Not Kv1.1 Potassium Channels: A Pore Region Determinant Dictates the Effect of Glycosylation on Trafficking. Journal of Biological Chemistry 279, 8879-8885, https://doi.org/10.1074/jbc.M309802200 (2004).

42. Zhu, J. et al. The Kv1.2 potassium channel: The position of an $\mathrm{N}$-glycan on the extracellular linkers affects its protein expression and function. Brain Research 1251, 16-29, https://doi.org/10.1016/j.brainres.2008.11.033 (2009).

43. Zhu, J., Yan, J. \& Thornhill, W. B. N-glycosylation promotes the cell surface expression of Kv1.3 potassium channels. FEBS Journal 279, 2632-2644, https://doi.org/10.1111/j.1742-4658.2012.08642.x (2012).

44. Watanabe, I. et al. Glycosylation Affects Rat Kv1.1 Potassium Channel Gating by a Combined Surface Potential and Cooperative Subunit Interaction Mechanism. The Journal of Physiology 550, 51-66, https://doi.org/10.1113/jphysiol.2003.040337 (2003).

45. Zhu, J., Watanabe, I., Gomez, B. \& Thornhill, W. B. Heteromeric Kv1 Potassium Channel Expression: Amino Acid Determinants Involved In Processing and Trafficking to the Cell Surface. Journal of Biological Chemistry 278, 25558-25567, https://doi. org/10.1074/jbc.M207984200 (2003). 
46. Sanguinetti, M. C. Dysfunction of delayed rectifier potassium channels in an inherited cardiac arrhythmia. Annals of the New York Academy of Sciences 868, 406-413 (1999).

47. Chiang, C. E. \& Roden, D. M. The long QT syndromes: genetic basis and clinical implications. Journal of the American College of Cardiology 36, 1-12 (2000).

48. Li, M., Jan, Y. N. \& Jan, L. Y. Specification of subunit assembly by the hydrophilic amino-terminal domain of the Shaker potassium channel. Science 257, 1225-1230 (1992).

49. Zhao, L. L., Qi, Z., Zhang, X. E., Bi, L. J. \& Jin, G. Regulatory role of the extreme C-terminal end of the S6 inner helix in C-terminaltruncated Kv1.2 channel activation. Cell biology international 34, 433-439, https://doi.org/10.1042/cbi20090009 (2010).

50. Balajthy, A. et al. 7DHC-induced changes of Kv1.3 operation contributes to modified T cell function in Smith-Lemli-Opitz syndrome. Pflugers Arch 468, 1403-1418, https://doi.org/10.1007/s00424-016-1851-4 (2016).

51. Attali, B. et al. Cloning, functional expression, and regulation of two $\mathrm{K}+$ channels in human $\mathrm{T}$ lymphocytes. J Biol Chem 267, 8650-8657 (1992).

52. Hamill, O. P., Marty, A., Neher, E., Sakmann, B. \& Sigworth, F. J. Improved patch-clamp techniques for high-resolution current recording from cells and cell-free membrane patches. Pfluegers Archiv - European Journal of Physiology 391(2), 85-100 (1981).

\section{Acknowledgements}

This work was supported by Mecenatura (P.H.), GINOP-2.3.2-15-2016-00044 (The project is co-financed by the European Union and the European Regional Development Fund) and NKFIH K119417 (G.P.). A.B. was cofinanced by Astellas Pharma Fellowship and National Excellence Program. P.H. is supported by the Bolyai János Fellowship. This manuscript was also supported by COST action BM 1406. The authors express their gratitude to Peter Nagy for his assistance in the watershed-segment analysis.

\section{Author Contributions}

O.V., O.S. and A.B. designed and performed the experiments, prepared the figures and revised the manuscript. S.S. and G.P. analyzed the data and revised the manuscript. P.H. analyzed the data, wrote the manuscript. All authors reviewed the manuscript.

\section{Additional Information}

Supplementary information accompanies this paper at https://doi.org/10.1038/s41598-018-24159-8.

Competing Interests: The authors declare no competing interests.

Publisher's note: Springer Nature remains neutral with regard to jurisdictional claims in published maps and institutional affiliations.

(c) (i) Open Access This article is licensed under a Creative Commons Attribution 4.0 International License, which permits use, sharing, adaptation, distribution and reproduction in any medium or format, as long as you give appropriate credit to the original author(s) and the source, provide a link to the Creative Commons license, and indicate if changes were made. The images or other third party material in this article are included in the article's Creative Commons license, unless indicated otherwise in a credit line to the material. If material is not included in the article's Creative Commons license and your intended use is not permitted by statutory regulation or exceeds the permitted use, you will need to obtain permission directly from the copyright holder. To view a copy of this license, visit http://creativecommons.org/licenses/by/4.0/.

(c) The Author(s) 2018 